\title{
Evaluación de los indicadores de impacto ambiental por el vertimiento de los efluentes industriales pesqueros en la Bahía de Coishco-Perú-2018
}

\author{
Bach. José Guevara del Aguila \\ jguevaradelaguila@gmail.com \\ Universidad Nacional Mayor de San Marcos, \\ Facultad de Ingeniería Geológica, Minería, Metalúrgica y Geográfica, \\ E.P Ingeniería Geológica. \\ Av. Venezuela s/n. Cuadra 34 Ciudad Universitaria, Lima-Perú
}

\section{RESUMEN}

La presente investigación tuvo como objetivo evaluar los indicadores de impacto ambiental por el vertimiento de los efluentes industriales pesqueros en el mar de la Bahía de Coishco-Perú, durante el período de febrero a noviembre del año 2018. El proceso metodológico empleó una investigación de tipo aplicada, enfoque cuantitativo, tuvo como diseño de investigación no experimental - explicativa, longitudinal. La muestra de estudio estuvo formada por 7 establecimientos industriales pesqueros. Para la recolección de información se utilizó resultados de monitoreos de efluentes y calidad de agua superficial, además se realizó visitas de campo. Como resultados se obtuvo, que al comparar los resultados promedio de las concentraciones de los parámetros de aceites y grasa (AyG) y sólidos suspendidos totales (SST), presentes en los efluentes con los Límites Máximos Permisibles (LMP) del sector pesca, señalan que las plantas que vierten en la zona submareal la gran mayoría cumplen con los LMP, distinta es la situación que se vive en la zona intermareal donde se observa excesos, siendo un indicador de falta de tratamiento de las aguas residuales.

Palabras clave: indicadores de impacto ambiental; indicadores de gran impacto; carga orgánica; efluentes industriales pesqueros; bahía de coishco 


\title{
Evaluation of environmental impact indicators for the discharge of industrial fishing effluents in Coishco Bay-Peru-2018
}

\begin{abstract}
The present research had the objective of evaluating the environmental impact indicators due to the discharge of industrial fishing effluents in the sea of the Bay of Coishco-Peru, during the period from February to November 2018. The methodological process employed an applied type research, quantitative approach, had as a non-experimental research design - explanatory, longitudinal. The study sample consisted of 7 industrial fishing establishments. For the collection of information, the results of effluent and surface water quality monitoring were used, and field visits were made. The results showed that when comparing the average results of the concentrations of oil and grease $(\mathrm{O} \& \mathrm{G})$ and total suspended solids (TSS) parameters present in the effluents with the Maximum Permissible Limits (MPL) of the fishing sector, the majority of the plants that discharge in the subtidal zone comply with the MPL; the situation is different in the intertidal zone where excesses are observed, which is an indicator of lack of wastewater treatment.
\end{abstract}

Keywords: environmental impact indicators; high impact indicators; organic load; industrial fishery effluents; coishco bay.

Artículo recibido: 20 diciembre. 2021

Aceptado para publicación: 10 enero 2022

Correspondencia: jguevaradelaguila@gmail.com

Conflictos de Interés: Ninguna que declarar 


\section{INTRODUCCIÓN}

Las aguas costeras de la Bahía de Coishco, reciben como lugar de disposición uno de los principales aspectos ambientales relacionado a los vertimientos de los efluentes industriales pesqueros provenientes de las actividades industriales pesqueras (UNS, 2009) dedicadas al procesamiento de consumo humano directo (congelado y enlatado) (Rosales, 2012) - (Rodríguez, 2007) e indirecto (Bereche, 2019) - (Miguel Angel, 2018) - (harina, aceite de pescado y harina residual), que llegan directamente e indirectamente al mar, dichas industrias se encuentran emplazadas en los distritos de Santa y Coishco. La bahía de Coishco, se ubica en un área aproximada de $11.625 \mathrm{~km}^{2}$ es una bahía abierta, rodeada por islas Santa y Moñaque, en sus profundidades predominan las isobatas desde 12 a 16 m y en el centro de la bahía existe alta concentración de materia orgánica. Los niveles de contaminación marina se han incrementado considerablemente, debido al aumento de la actividad pesquera que se desarrolla en la zona y genera aguas residuales con alto contenido orgánico. También el incremento de la contaminación se debe a los vertimientos urbanos y aguas de escorrentía agrícola, tal como la anoxia determinada en mayo de 2004 (Tresierra, et al, 2007). La bahía de Coishco es una zona importante en el desarrollo industrial regida por el abastecimiento de los recursos hidrobiológicos, dicho abastecimiento se realiza de manera diaria tomando en cuenta las actividades de consumo humano directo que no se encuentran sujetas a periodos de veda y de manera cíclica o temporal en los periodos de pesca correspondientes al procesamiento del recurso hidrobiológico anchoveta - Engraulis ringens en la elaboración de harina y aceite de pescados. Durante los años 2015 y 2016 (Aquino \& Maguiña); dieron a conocer la magnitud de la contaminación marina producida por los efluentes industriales en la bahía de Coischo en los periodos de pesca y veda.

El Estudio de Impacto ambiental realizado por el profesor Valverde Bustamante, J. H. (2014) para la planta pesquera Sálica en el Ecuador, dedicada al enlatado de productos hidrobiológicos, identificó los impactos ambientales generados por las descargas de aguas residuales y propuso un sistema que permite controlar sus impactos en la zona influencia. Por su parte, el profesor Romero (2013), en su estudio "Aporte contaminante del procesamiento de recursos pesqueros en Cuba y su impacto al medio", expresa que los aportes de contaminantes al medio acuático por concepto de materia prima procesada en 
instalaciones pesqueras cubanas eran principalmente: nitrógeno total, fosforo total y nitrógeno amoniacal.

Desde la posición de Esquivel \& Yalico (2015), concluyeron que la contaminación de las aguas costeras de la Bahía de Chancay es ocasionada por el vertido de efluentes residuales de la industria pesquera.

Cabrera (2002) señalo en su estudio de la contaminación de las aguas costeras de la Bahía de Chancay, concluyó que el agua de mar de la Bahía de Chancay presentaba valores que están muy por encima de las normas vigentes (Ley de Aguas),

Altaner (2009) indica que el efluente residual evacuado de las instalaciones del establecimiento industrial, con destino directo a los sistemas de recolección de aguas servidas o cuerpos receptores pueden ser todo curso o masa de agua, natural o artificial, superficial o subterráneo.

Por su parte, el Banco Mundial (2007) señala que las aguas residuales procedentes del procesamiento de pescado tienen un alto contenido orgánico y por consiguiente una elevada Demanda Bioquímica de Oxígeno (DBO), debido a la presencia de sangre, tejidos y proteínas disueltas. Además, tiene un elevado contenido en nitrógeno (sobre todo cuando hay sangre presente) y fósforo. También puede haber detergentes y desinfectantes presentes en las corrientes de aguas residuales después de su aplicación durante las actividades de limpieza en las instalaciones, empleando diversas sustancias químicas, como detergentes, ácidos, alcalinos, neutros y desinfectantes que ocasionan problemas serios de contaminación. En el año 2000 Sánchez, detalla que la contaminación por efluentes pesqueros es la más impactante entre las fuentes de origen industrial, causando una serie alteración de los parámetros indicadores de la calidad del medio marino, especialmente en bahía semicerradas de aguas someras, como es el caso de la bahía de Paracas. Los efectos de los efluentes industriales pesqueros descargados en bahías como por ejemplo, el Ferrol conteniendo altas concentraciones de materia orgánica, ocasionó que grandes áreas del fondo fueran tapizadas, causando serios problemas, por interferencia en la relación agua sedimento, impidiendo el desarrollo de toda forma de vida, y favoreciendo el desarrollo de la flora microbiana anaeróbica, y con ello la generación de sustancias nocivas para la vida, como sulfuros, amonio, etc., que son perjudiciales al ecosistema acuático (CONAM, 1999).

Los efluentes líquidos generados del procesamiento de pescado que son vertidos a 
cuerpos hídricos receptores pueden provocar efectos (i) inmediatos, a mediano plazo y a largo plazo Ambrosio, M.J. (2004),

En el Perú existe poca información que sustente como los volúmenes descargas de efluentes industriales, afectan la calidad del agua de mar, los monitoreos solo se enfocan en determinar el estado del cuerpo de agua producto de las actividades antrópicas.

\section{ESTRATEGIAS METODOLÓGICAS O MATERIALES Y MÉTODOS}

\subsection{Tipo y diseño de investigación}

Según el tipo de abstracción, la investigación es aplicada, porque propone un fin de recabar información de resultados de monitoreos de efluentes y hacer comparativos con los LMP del sector pesca, asimismo, estimar el volumen de efluentes generados en relación a la recepción de la materia prima por actividad de procesamiento industrial pesqueros, dicha información de recepción se solicitó al Ministerio de la Producción, igualmente la carga contaminante se consideró en virtud a los resultados promedio de la concentración correspondiente al monitoreo de efluentes industriales de siete (7) establecimientos industriales pesqueros y evaluar la calidad del agua superficial, doce (12) puntos de monitoreo donde se compara la calidad del agua superficial, dicha información se requirió al Organismo de Evaluación y Fiscalización Ambiental.

Según temporalidad, longitudinal, ya que recolecta datos a través del tiempo en lugares y puntos específicos, con la finalidad de identificar cambios en las variables de estudio encontrados en el mar de la bahía de Coishco.

Según su naturaleza de datos, metodología cuantitativa, porque recolecta datos de resultados físicos, químicos y microbiológicos de los efluentes y calidad de agua de mar en la bahía de Coishco, durante el periodo de febrero a noviembre del 2018. Para probar una hipótesis con base en la relación numérica y análisis estadístico.

Según manipulación de las variables, investigación no experimental, porque no hay manipulación de las variables, estas se observan y describen tal como se presentan en los resultados.

Según la naturaleza de los objetivos, investigación explicativa porque persigue describir o acercarse al problema, y además intenta encontrar las causas del mismo.

\subsection{Unidad de análisis}

Comprende las concentraciones y cargas contaminantes presente en los efluentes industriales pesqueros de siete (7) establecimientos industriales las que tendrán la 
siguiente denominación: (i) Planta A, (ii) Planta B, (iii) Planta C, (iv) Planta D, (v) Planta E, (vi) Planta F y (vii) Planta G que son evacuados al agua de más de la bahía de Coishco, igualmente los once (11) puntos de toma de agua de mar se tomaron en áreas donde se desarrolla la actividad procesamiento pesquero industrial y un (1) punto sin influencia de la actividad, en el que se evaluará los parámetros físicos y químicos serán comparados con los estándares de calidad ambiental ECA-Agua en la bahía.

2.3. Población de estudio: Las concentraciones y cargas contaminantes presentes en los efluentes y las características físico, químicas y microbiológicas del agua superficial, donde se dispersa el vertimiento de efluentes industriales pesqueros en la bahía de Coishco-Perú.

2.4. Tamaño de muestra: El tamaño de la muestra está en función al área donde posiblemente influya el vertimiento de los efluentes industriales pesqueros, de siete establecimientos industriales pesqueros dedicados al procesamiento de harina y aceite de pescado, enlatado, congelado y harina residual. Las clasificaciones de los puntos se han establecido en función al Protocolo Nacional para el Monitoreo de la Calidad de los Recursos Hídricos Superficiales-Resolución Jefatural Nº 010-2016-ANA, los puntos se señalan a continuación:

Tabla 1. Puntos de Monitoreo en el mar de la Bahía de Coishco.

\begin{tabular}{|c|c|c|c|c|c|}
\hline \multirow{2}{*}{$\begin{array}{l}\text { Puntos de } \\
\text { muestreo }\end{array}$} & \multirow{2}{*}{ Descripción } & \multirow{2}{*}{ Código } & \multicolumn{2}{|c|}{ Coordenadas } & \multirow{2}{*}{\begin{tabular}{|c|}
$\begin{array}{c}\text { Componente } \\
\text { ambiental }\end{array}$ \\
Agua \\
Superficie
\end{tabular}} \\
\hline & & & Norte & Este & \\
\hline \multirow{4}{*}{$\begin{array}{c}\text { Zona } \\
\text { intermareal }\end{array}$} & \multirow{4}{*}{$\begin{array}{l}\text { Orilla de } \\
\text { Playa }\end{array}$} & AM-CO1 & 9002073.00 & 761288.00 & $\mathrm{X}$ \\
\hline & & AMCO2 & 9002708.00 & 761164.00 & $X$ \\
\hline & & AM-CO3 & 9002946.00 & 761006.00 & $X$ \\
\hline & & AM-CO4 & 9003369.00 & 760615.00 & $X$ \\
\hline \multirow{7}{*}{$\begin{array}{c}\text { Zona } \\
\text { submareal }\end{array}$} & \multirow{7}{*}{$\begin{array}{l}\text { Cercanos a } \\
\text { chata y } \\
\text { emisarios } \\
\text { submarinos }\end{array}$} & AM-CO5 & 9002043.00 & 760768.00 & $\mathrm{X}$ \\
\hline & & AM-CO6 & 9002163.00 & 760751.00 & $\mathrm{X}$ \\
\hline & & AM-CO7 & 9002237.00 & 760735.00 & $X$ \\
\hline & & AM-CO8 & 9002316.00 & 760520.00 & $X$ \\
\hline & & AM-CO9 & 9002518.00 & 760525.00 & $X$ \\
\hline & & AM-CO10 & 9001871.00 & 760141.00 & $X$ \\
\hline & & AM-CO11 & 9002331.00 & 760267.00 & $X$ \\
\hline $\begin{array}{l}\text { Zona en blanco } \\
\text { (Submareal) }\end{array}$ & $\begin{array}{l}\text { Aguas } \\
\text { afuera }\end{array}$ & AM-CO12 & 9000319.00 & 757489.00 & $X$ \\
\hline
\end{tabular}

Fuente: Elaboración propia 
Figura 1. Ubicación de los puntos de muestro en la zona intermareal de la Bahía de Coishco.

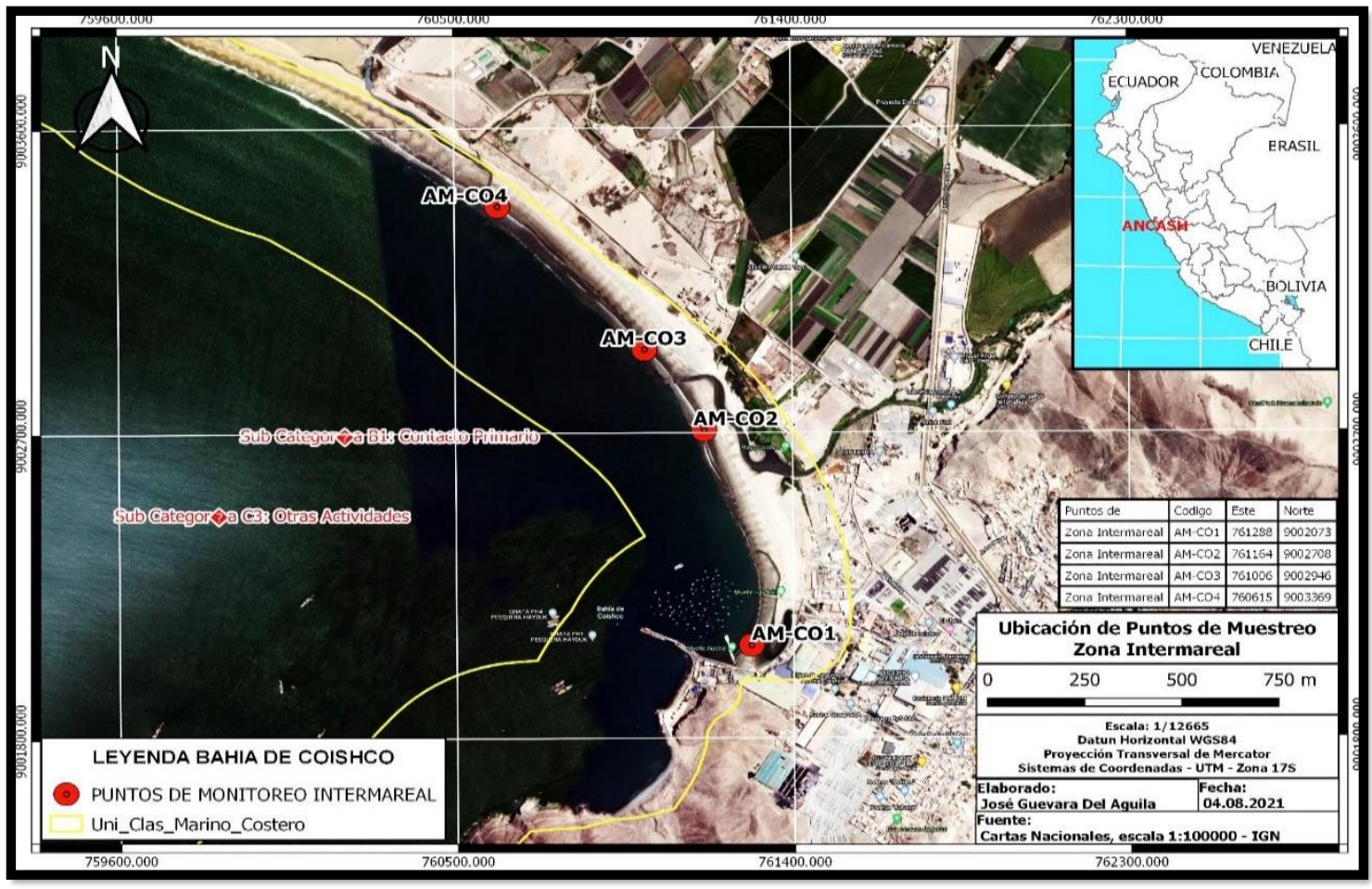

Figura 2. Ubicación de los puntos de muestro en la zona Submareal de la Bahía de Coishco

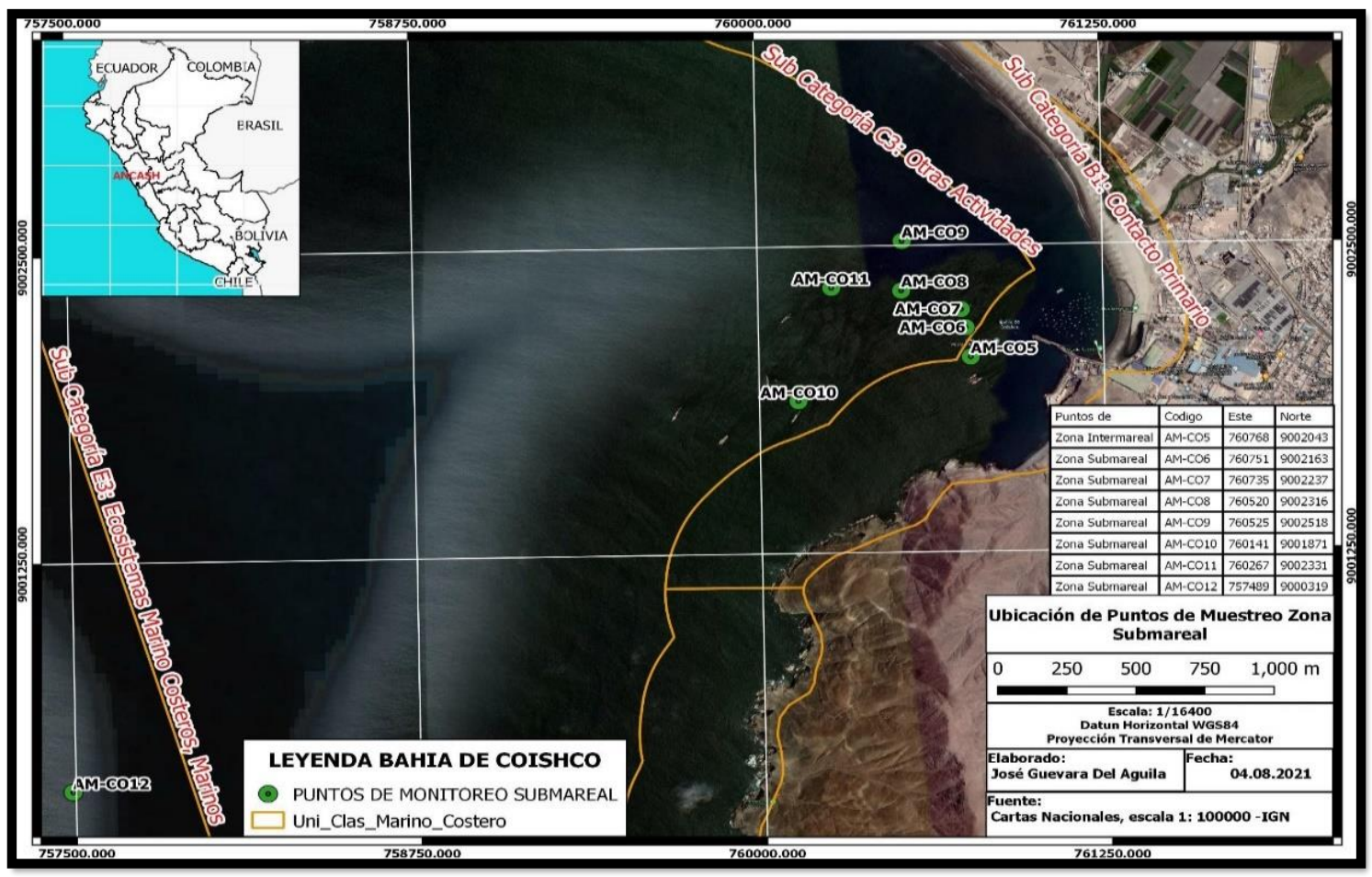




\subsection{Técnicas de recolección de datos}

Para la recopilación de información, se requirió información de resultados de monitoreo de efluentes y calidad de agua superficial, asimismo recepción de materia prima (Austin, 2013), se realizó visitas de campo, análisis documental, se utilizó técnicas para asegurar la confiabilidad, validez y objetividad de los resultados.

\subsection{Parámetros y métodos de análisis}

a) Para la evaluación de efluentes: Los parámetros estuvieron en función a la Resolución ministerial Nº61-2016-PRODUCE. Para el análisis del nivel de carga orgánica se utilizó parámetros Sólidos Suspendidos Totales, Demanda Bioquímica de Oxígeno, Demanda Química de Oxígeno y Aceites y grasas, ello debido a que la unidad de expresión mg/L que ayuda a determinar las cargas en función a un volumen determinado de evaluación de efluentes.

A continuación, en la tabla 2 se describen los parámetros y métodos de análisis y el laboratorio que lo realizó.

Tabla 2. Parámetros evaluados y métodos de análisis según laboratorio para los efluentes

\begin{tabular}{|c|c|c|c|c|}
\hline $\mathbf{N}^{\circ}$ & Parámetros & Unidad & Método de análisis & $\begin{array}{c}\text { Laboratorio de } \\
\text { ensayo }\end{array}$ \\
\hline 1 & $\begin{array}{l}\text { Demanda } \\
\text { Bioquímica de } \\
\text { Oxígeno }\end{array}$ & $\mathrm{mg} / \mathrm{L}$ & $\begin{array}{l}\text { APHA_AWWA-WPCF } \\
\text { Estándar Methods for waste } \\
\text { wáter. } \\
\text { Part5210B, 22nd SWEWW- } \\
\text { APHA-AWWA-WEF.Part } \\
\text { 5210B, 22nd ED.2012, } \\
\text { Biochemical } \\
\begin{array}{l}\text { Demand (BOD) } 5 \text { day Test. } \\
\text { ED.2012. }\end{array}\end{array}$ & \\
\hline 2 & $\begin{array}{l}\text { Sólidos } \\
\text { Suspendidos } \\
\text { Totales }\end{array}$ & $\mathrm{mg} / \mathrm{L}$ & $\begin{array}{l}\text { SMEWW-APHA-AWWA- } \\
\text { WEF.Part.2540D, 22nd } \\
\text { Ed.2012. Standard Methods } \\
\text { For Examination of Water and } \\
\text { Wastewater. Solids Total } \\
\text { Supended Dried at } 103-105^{\circ} \mathrm{C}\end{array}$ & $\begin{array}{l}\text { Colecbi S.A.C } \\
\text { ALS S.A.C }\end{array}$ \\
\hline 3 & $\begin{array}{l}\text { Aceites } \\
\text { Grasas }\end{array}$ & $\mathrm{mg} / \mathrm{L}$ & $\begin{array}{l}\text { SWEWW-APHA-AWWA- } \\
\text { WEF.Part.5520D, 22nd, } \\
\text { Ed.2012. Estándar Methods } \\
\text { For Examination of Water and } \\
\text { Wastewater. Oil and Grease. } \\
\text { Soxhlet extraction Mehod }\end{array}$ & \\
\hline
\end{tabular}




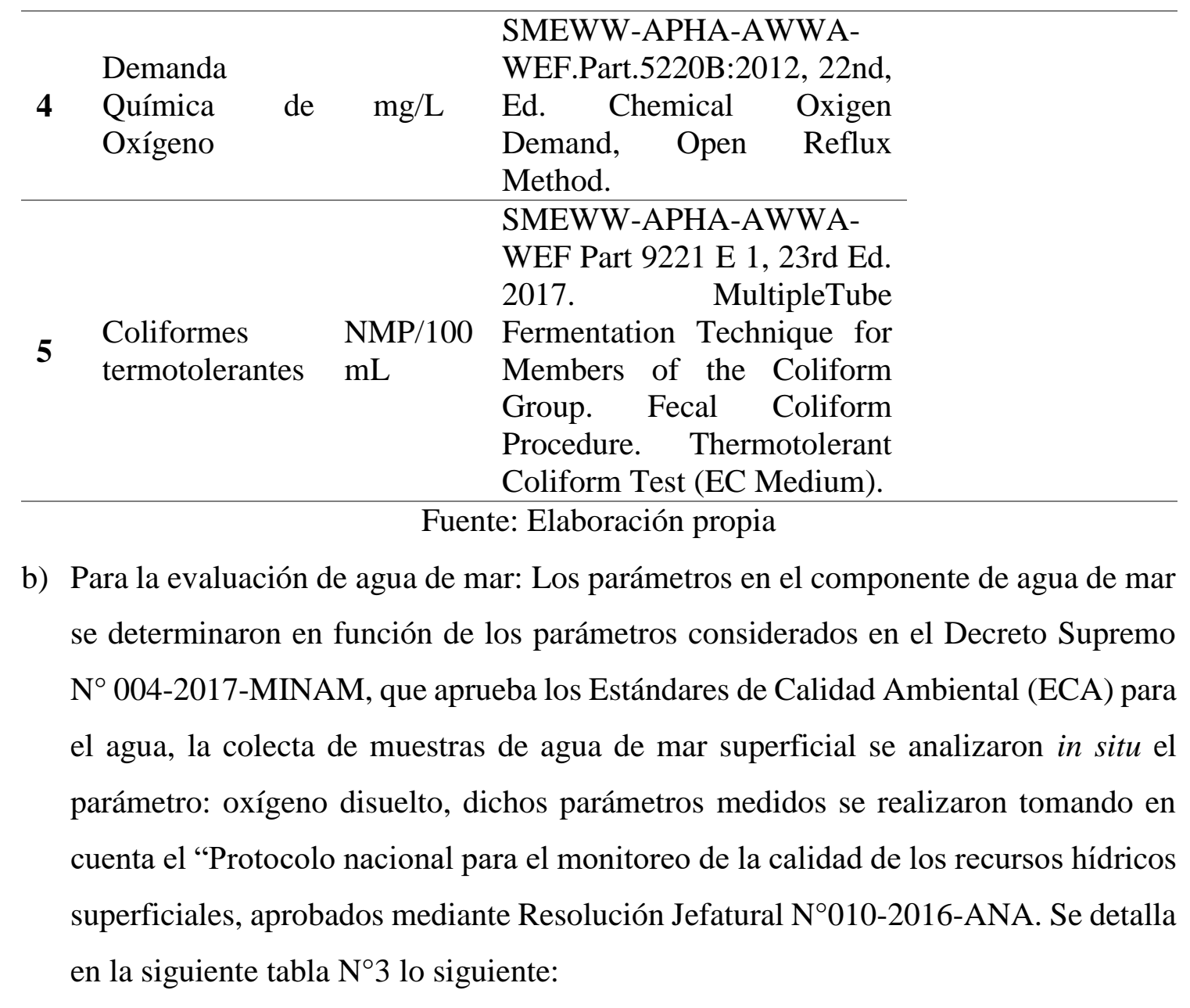

Tabla 3. Parámetros evaluados y métodos de análisis según laboratorio para el agua de mar superficial

\begin{tabular}{|c|c|c|c|c|}
\hline $\mathbf{N}^{\circ}$ & Parámetros & Unidad & Método de análisis & $\begin{array}{c}\text { Laboratorio } \\
\text { de ensayo }\end{array}$ \\
\hline 1 & Aceites y Grasas & $\mathrm{mg} / \mathrm{L}$ & $\begin{array}{ll}\text { ASTM } & \text { D7066-04 } \\
\text { (Validado), } 2011 & \end{array}$ & \\
\hline 2 & $\begin{array}{l}\text { Demanda } \\
\text { Bioquímica } \\
\text { Oxígeno }\end{array}$ & $\mathrm{mg} / \mathrm{L}$ & $\begin{array}{l}\text { SMEWW-APHA-AWWA- } \\
\text { WEF Part } 5210 \quad \text { B， 23rd } \\
\text { Ed.2017 }\end{array}$ & \\
\hline 3 & $\begin{array}{l}\text { Demanda Química } \\
\text { de Oxígeno }\end{array}$ & $\mathrm{mg} / \mathrm{L}$ & $\begin{array}{l}\text { SMEWW-APHA-AWWA- } \\
\text { WEF Part } 5210 \text { B, 23rd Ed. } \\
2017\end{array}$ & ALS S.A.C \\
\hline 4 & $\begin{array}{l}\text { Sólidos Totales } \\
\text { Suspendidos }\end{array}$ & $\mathrm{mg} / \mathrm{L}$ & $\begin{array}{l}\text { SMEWW-APHA-AWWA- } \\
\text { WEF Part } 2540 \text { D, 22nd Ed. } \\
2012\end{array}$ & \\
\hline 5 & $\begin{array}{l}\text { Coliformes } \\
\text { termotolerantes }\end{array}$ & $\begin{array}{l}\mathrm{NMP} / 100 \\
\mathrm{~mL}\end{array}$ & $\begin{array}{l}\text { SMEWW-APHA-AWWA- } \\
\text { WEF Part } 9221 \text { E } 1,23 \\
\text { Ed.2007 }\end{array}$ & \\
\hline
\end{tabular}

Fuente: Elaboración propia 


\section{RESULTADOS Y DISCUSIÓN}

\subsection{Resultados de acuerdo al Objetivo 1: Comparar el nivel de concentración de los} parámetros físico, químicos y microbiológicos de los efluentes industriales pesqueros que sobrepasan los LMP del sector industrial y PTAR doméstica en la Bahía de Coishco-Perú, durante el periodo de febrero a noviembre del año 2018.

a) Resultados de los valores de los LMP que vierten en la zona submareal

Tabla $\mathbf{N}^{\circ} \mathbf{4}$ Resultados promedio de valores comparados con los LMP de aquellas plantas que vierten en la zona submareal.

\begin{tabular}{llccccccc}
\hline \multicolumn{1}{c}{ Parámetro } & \multicolumn{1}{c}{ Sector } & LMP & $\begin{array}{c}\text { Valor } \\
\text { mínimo }\end{array}$ & $\begin{array}{c}\text { Valor } \\
\text { máximo }\end{array}$ & Planta A & $\begin{array}{c}\text { Planta } \\
\text { B }\end{array}$ & $\begin{array}{c}\text { Planta } \\
\text { C }\end{array}$ & $\begin{array}{c}\text { Plant } \\
\text { a D }\end{array}$ \\
\hline AyG $(\mathrm{mg} / \mathrm{L})$ & Pesca & 350 & 7.67 & 69.44 & 69.44 & 96.44 & 66.56 & 7.67 \\
\hline SST $(\mathrm{mg} / \mathrm{L})$ & Pesca & 700 & 26.33 & 1223.33 & 272.16 & 316.56 & $\mathbf{1 2 2 3 . 3 3}$ & 26.33 \\
\hline $\mathrm{pH}$ & Pesca & $5-9$ & 5.40 & 7.89 & 6.03 & 6.01 & 5.40 & 7.89 \\
\hline DBO (mg/L) & PTAR & 100 & 73.33 & 4803.29 & $\mathbf{2 2 4 9 . 0 0}$ & $\mathbf{2 9 0 5 . 3 9}$ & $\mathbf{4 8 0 3 . 2 9}$ & 73.33 \\
& doméstica & & & & & & & \\
\hline DQO (mg/L) & PTAR & 200 & 117.33 & 6987.33 & $\mathbf{2 1 3 3 . 0 6}$ & $\mathbf{3 2 9 6 . 0 0}$ & $\mathbf{6 9 8 7 . 3 3}$ & 117.33 \\
& doméstica & & & & & & & \\
\hline C.T (NMP/100 & PTAR & 10000 & 129.67 & 6913413.33 & $\mathbf{1 2 9 . 6 7}$ & $\mathbf{6 9 1 3 4 1 3 . 3 3 ~ 8 5 2 9 2 6 . 6 7}$ & 163.33 \\
$\mathrm{~mL})$ & doméstica & & & & & &
\end{tabular}

\section{Fuente: Elaboración propia}

- Aceites y grasas (AyG): Los valores promedio obtenidos cumplen con los LMP del sector pesca siendo el valor mínimo 7,67 mg/L y el valor máximo 96,44 mg/L.

- Sólidos suspendidos totales (SST): Se observa que, de las 4 plantas, una excede los LMP reportando un valor del promedio del periodo evaluado de $1223,33 \mathrm{mg} / \mathrm{L}$ lo que representa un exceso de $74,76 \%$, representando una posible deficiencia en el sistema de tratamiento para la reducción del parámetro.

- $\mathrm{pH}$ : En lo referente a valores promedio del parámetro $\mathrm{pH}$, ninguna planta excedió los LMP, siendo los resultados con tendencia a la acidez de 5,40 y de alcalinidad con 7,89 respectivamente.

- Demanda bioquímica de oxígeno (DBO): Este parámetro no cuenta con LMP para el sector, debido a ese alcance se utilizó de manera referencial los LMP de PTAR, los resultados fueron excedentes para tres plantas, siendo solo una la que cumple con el límite referencial, siendo el valor mínimo de $117.33 \mathrm{mg} / \mathrm{L}$ y valor máximo 6987.33 $\mathrm{mg} / \mathrm{L}$. 
- Coliformes termotolerantes (CT): Este parámetro microbiológico mostró un comportamiento donde dos (2) plantas superan el LMP referencial y dos (2) están por debajo. Siendo el valor mínimo de 129.67 NMP/100 mL y el valor máximo de 6913413.33 NMP/100 mL, estos resultados pueden deberse a falta de buenas prácticas de saneamiento por parte de las plantas, captación de agua para sus procesos con alta presencia de Coliformes, asimismo el sistema de tratamiento con los que cuentan las plantas no cumple la función de reducir y/o eliminar este parámetro.

b) Resultados de los valores promedio de los LMP que vierten en la zona intermareal Al igual que en la zona de vertimiento submareal se utilizarán parámetros del sector pesca y de la PTAR doméstica para comparar los resultados promedios de los efluentes industriales pesqueros, las características de estas plantas E, F y G es que vierten de manera indirecta sus efluentes industriales pesqueros a la bahía de Coishco siendo su medio de traslado la quebrada el Cascajal, los resultados del periodo se detallan en la tabla $\mathrm{N}^{\circ} 5$.

Tabla $\mathbf{N}^{\circ} 5$ Resultados promedio de valores comparados con los LMP de aquellas plantas que vierten en la zona intermareal

\begin{tabular}{llcccccc}
\hline Parámetro & Sector & $\begin{array}{c}\text { Valor } \\
\text { mínimo }\end{array}$ & $\begin{array}{c}\text { Valor } \\
\text { máximo }\end{array}$ & $\begin{array}{c}\text { LMP } \\
\text { Planta }\end{array}$ & $\begin{array}{c}\text { Planta } \\
\text { E }\end{array}$ & $\begin{array}{c}\text { Planta } \\
\text { G }\end{array}$ \\
\hline SST $(\mathrm{mg} / \mathrm{L})$ & Pesca & 151.19 & 9997.00 & $\mathbf{3 5 0}$ & $\mathbf{1 5 0 4 . 4 8}$ & 151.19 & $\mathbf{9 9 9 7 . 0 0}$ \\
\hline pH & Pesca & 453.00 & 8634.89 & $\mathbf{7 0 0}$ & $\mathbf{6 4 3 9 . 4 4}$ & 453.00 & $\mathbf{8 6 3 4 . 8 9}$ \\
\hline DBO $(\mathrm{mg} / \mathrm{L})$ & Pesca & 6.70 & 7.12 & $\mathbf{5 - 9}$ & 6.70 & 7.12 & 6.80 \\
& PTAR & 1599.43 & 25611.00 & $\mathbf{1 0 0}$ & $\mathbf{1 4 7 7 6 . 5 6}$ & $\mathbf{1 5 9 9 . 4 3}$ & $\mathbf{2 5 6 1 1 . 0 0}$ \\
& doméstica & & & & & & \\
\hline DQO $(\mathrm{mg} / \mathrm{L})$ & PTAR & 2178.67 & 19343.33 & $\mathbf{2 0 0}$ & $\mathbf{1 9 3 4 3 . 3 3}$ & $\mathbf{2 1 7 8 . 6 7}$ & $\mathbf{4 5 3 3 . 3 3}$ \\
& doméstica & & & & & & \\
\hline C.T $(\mathrm{NMP} / 100$ & PTAR & 1787500.00 & 82666666 & $\mathbf{1 0 0 0 0}$ & $\mathbf{8 2 6 6 6 6 6 6 . 6 7}$ & $\mathbf{1 7 8 7 5 0 0 . 0 0}$ & \\
$\mathrm{mL})$ & doméstica & & & & & & \\
\hline
\end{tabular}

Fuente: Elaboración propia

- Aceites y grasas (AyG): En este parámetro se puede observar un exceso en el promedio de resultados de LMP para dos (2) plantas del $329.85 \%$ y $2756.28 \%$, lo que podría deberse a una falta de tratamiento del parámetro.

- Sólidos suspendidos totales (SST): Lo observado señala que al igual que las grasas han sufrido un exceso de los LMP en dos (2) plantas de $813.92 \%$ y $1133.55 \%$, estos excesos son indicadores de una falta de tratamiento del parámetro SST. 
- pH: Los valores promedio del parámetro reflejan que ninguna planta excedió los LMP, siendo los resultados con tendencia a la acidez de 6.70 y de alcalinidad con 7,12 respectivamente.

- Demanda bioquímica de oxígeno (DBO): Se indicó que en la zona submareal la DBO, no cuenta con valor para los LMP del sector pesca se referenció con los LMP de PTAR doméstica, donde se obtuvo que de las tres (3) plantas evaluadas, las tres exceden los LMP, siendo el valor mínimo $1599.43 \mathrm{mg} / \mathrm{L}$ y un valor máximo 25611.00 $\mathrm{mg} / \mathrm{L}$.

- Demanda química de oxígeno (DQO): De manera referencial los valores promedio comparados con los LMP de PTAR domésticas, se obtuvo un exceso del promedio de concentración superior al LMP en las tres (3) plantas siendo el valor mínimo de $2178.67 \mathrm{mg} / \mathrm{L}$ y $19343.33 \mathrm{mg} / \mathrm{L}$.

- Coliformes termotolerantes (CT): Para este caso de las tres (3) plantas solo dos ejecutaron el análisis de este parámetro, de los reportado se puede concluir que ambos casos superan los LMP referenciales de PTAR doméstica siendo los valores de 82666666.67 NMP/100 mL y $1787500.00 \mathrm{~mL}$, lo que sería un indicador que los sistemas de tratamiento que poseen ambas plantas que no cumplen la finalidad de reducir este parámetro contaminante.

\title{
3.2. Resultados de acuerdo al objetivo 2: Determinar el nivel de carga contaminante
} presente en los efluentes industriales pesqueros del proceso que son vertidos al agua de mar de la Bahía de Coishco-Perú, durante el periodo de febrero a noviembre del año 2018.

Para el presente análisis se tomará en consideración los volúmenes de efluentes generados por un periodo de tiempo determinado y el valor de concentración reportado de los informes de ensayo. Dicha expresión citando del Ideam (2017) se encuentra contemplada en la siguiente fórmula:

\author{
$[$ Cc $(\mathrm{mg} / \mathrm{d})=($ Co $\mathrm{mg} / \mathrm{L}) \times($ Ca L/periodo $)]$ \\ Cc: Carga contaminante \\ Co: Concentración \\ Ca: Caudal
}


Para el respectivo diagnóstico se estimó el volumen de efluentes de siete (7) establecimientos industriales pesqueros (EIP) a los cuales se les denominó planta A, planta $\mathrm{B}$, planta $\mathrm{C}$, planta $\mathrm{D}$, planta $\mathrm{E}$ y planta $\mathrm{G}$, asimismo, con sus respectivos informes de ensayo se promedió y se estimó la carga contaminante de cada EIP que vierte directa e indirectamente a la bahía de Coishco en función a los parámetros $\left(\mathrm{DBO}_{5}\right),(\mathrm{SST})$, (DQO) y (Ay G).

a) Resultado del valor de carga orgánica evacuada en la zona submareal

Tabla $\mathbf{N}^{\circ} 6$ Volumen expresado en toneladas de carga orgánica evacuados por planta zona submareal de la Bahía de Coishco.

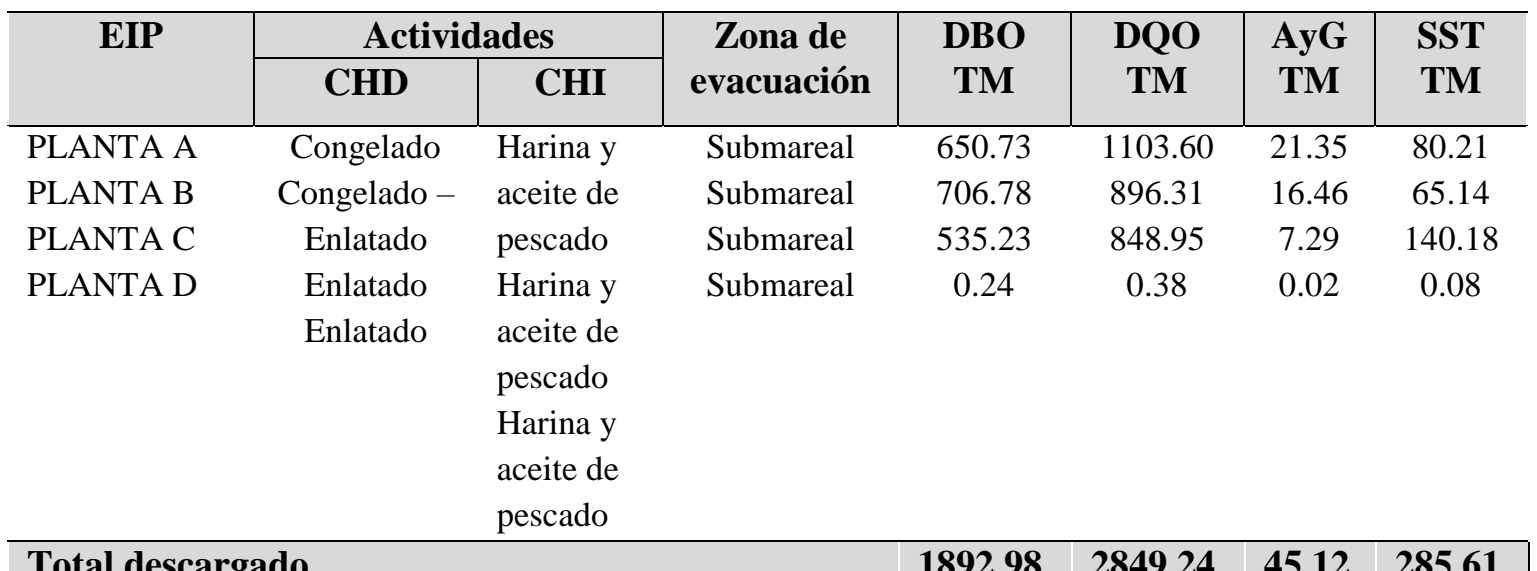

Fuente: Elaboración propia

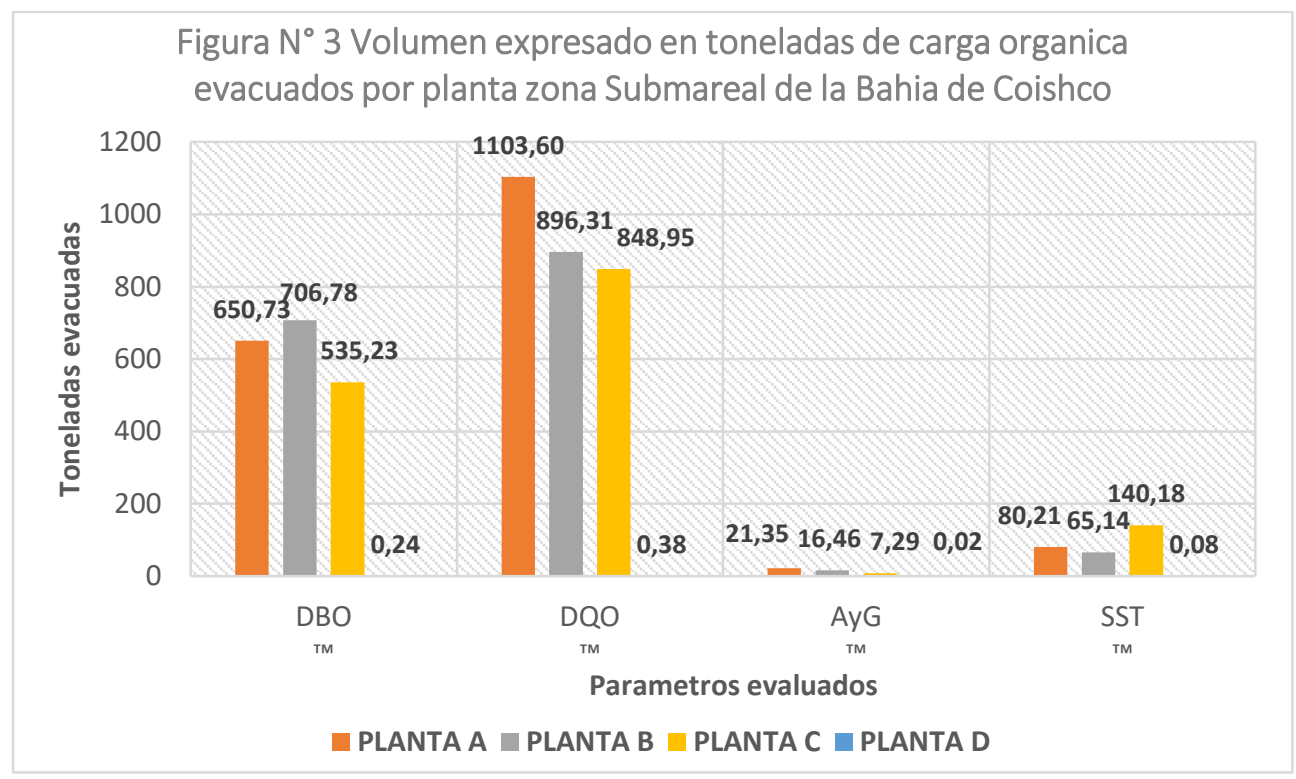

Fuente: Elaboración propia

Respecto a parámetro $\mathrm{DBO}_{5}$ y DQO (figura 3), las plantas con actividades de combinadas de enlatado, congelado y harina y aceite de pescado son las que evacuan mayor volumen 
de esta carga contaminante, siendo la planta B con 706.78 tm de DBO y planta A con 1103.60 tm de DQO. En los AyG, los volúmenes de evacuación en tm se encuentran en $21.56 \mathrm{tm}, 23.43 \mathrm{tm}, 7.39 \mathrm{tm}, 0.02 \mathrm{tm}$, para las plantas A, B, C y D estos rangos de evacuación demuestran que los sistemas de tratamiento de efluentes utilizados para recuperar las grasas son aprovechados en la reinserción del aceite.

En relación a los SST, las plantas que tiene actividades conjuntas de enlatado y harina y aceite de pescado presentan los mayores volúmenes de evacuación de SST, siendo la planta $\mathrm{C}$ la que muestra $140.18 \mathrm{tm}$ presentes en los efluentes, este resultado puede deberse al volumen de efluentes generados durante el periodo evaluado y a la presencia de concentraciones altas, esto debido al deficiente tratamiento de los efluentes.

b) Resultado del valor de carga orgánica evacuada en la zona intermareal

Tabla $\mathbf{N}^{\circ} \mathbf{7}$ Volumen expresado en toneladas de carga orgánica evacuados por planta zona intermareal de la Bahía de Coishco

\begin{tabular}{|c|c|c|c|c|c|c|c|}
\hline \multirow[t]{2}{*}{ EIP } & \multicolumn{2}{|c|}{ Actividades } & \multirow{2}{*}{$\begin{array}{c}\text { Zona de } \\
\text { evacuación }\end{array}$} & \multirow{2}{*}{$\begin{array}{c}\text { DBO } \\
\text { TM }\end{array}$} & \multirow{2}{*}{$\begin{array}{c}\text { DQO } \\
\text { TM }\end{array}$} & \multirow{2}{*}{$\begin{array}{c}\text { AyG } \\
\text { TM }\end{array}$} & \multirow{2}{*}{$\begin{array}{l}\text { SST } \\
\text { TM }\end{array}$} \\
\hline & CHD & CHI & & & & & \\
\hline PLANTA E & Enlatado & Harina & Intermareal & 63.78 & 83.50 & 6.49 & 27.80 \\
\hline PLANTA F & Enlatado & residual & Intermareal & 39.61 & 46.35 & 5.20 & 12.35 \\
\hline PLANTA G & Enlatado & $\begin{array}{l}\text { Harina } \\
\text { residual } \\
\text { Harina } \\
\text { residual }\end{array}$ & Intermareal & 253.07 & 44.79 & 98.78 & 85.32 \\
\hline
\end{tabular}

Total descargado

$\begin{array}{llll}356.46 & 174.64 & 110.47 & 125.47\end{array}$

Fuente: Elaboración propia

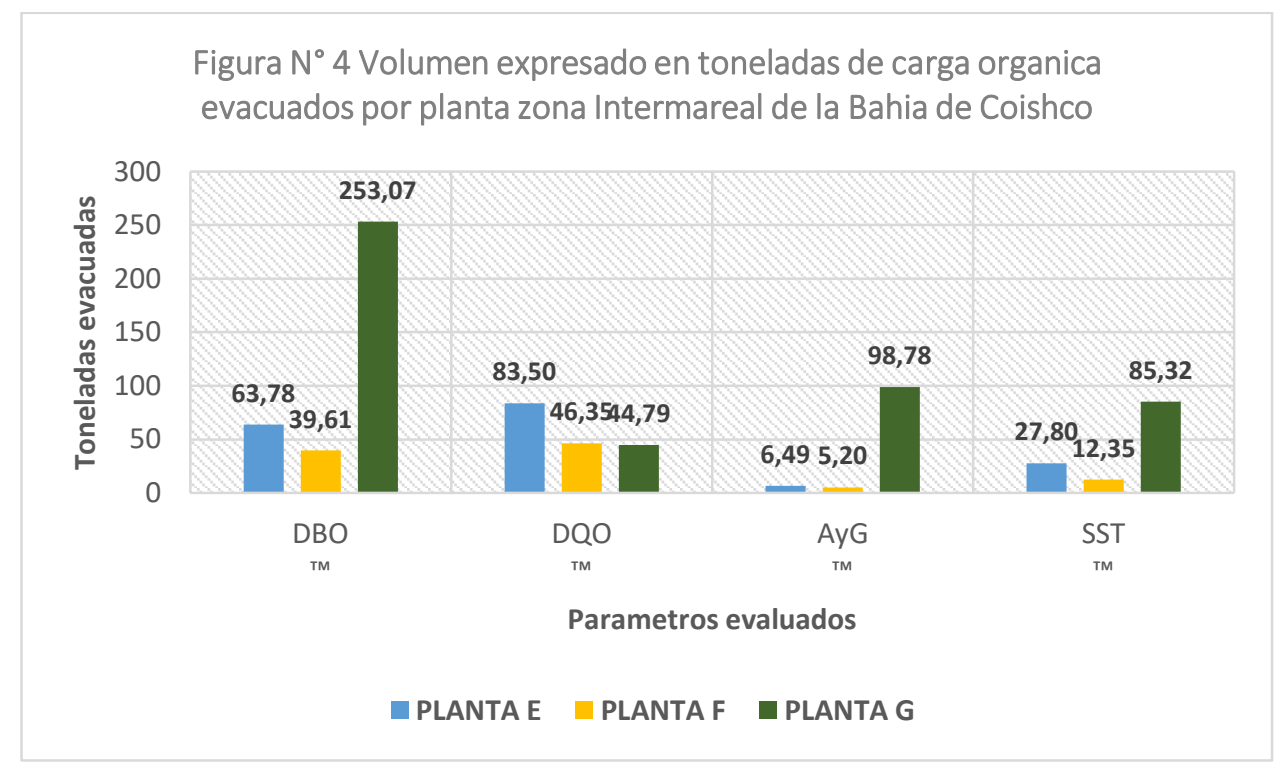

Fuente: Elaboración propia 
El parámetro DBO, reportaron volúmenes de $63.78 \mathrm{tm}, 39.61$ y $253.07 \mathrm{tm}$ correspondientes a las plantas $\mathrm{E}, \mathrm{F}$ y $\mathrm{G}$, siendo el mayor resultado presentado en la planta G, evacuado de manera indirecta a la Bahía de Coishco a través de la quebrada Cascajal. La DQO, los resultados estimados para el periodo son de $83.50 \mathrm{tm}, 46.35 \mathrm{tm}$ y $44.79 \mathrm{tm}$. En lo que respecta al parámetro AyG (Ver figura4), los volúmenes de evacuación se encuentran en $27.80 \mathrm{tm}$ y $12.35 \mathrm{tm}$ para las plantas E y F, sin embargo, la planta $\mathrm{G}$ presenta el mayor resultado de 98.74 tm de A y G presente en los efluentes.

La presencia de SST es baja debido a los días de producción y a los volúmenes de efluentes generando siendo la evacuación expresada en toneladas de $27.08 \mathrm{tm}$ y $12.35 \mathrm{tm}$, para las plantas E y F, sin embargo, para la planta G el volumen se encuentra en 85.32 tm, este resultado se observa en la tabla $\mathrm{N}^{\circ} 7$.

\section{c) Vertimiento de volumen en toneladas fuera rango permitido de los parámetros contaminantes que cuentan con LMP}

Se utilizaron valores de las concentraciones únicamente de los efluentes del proceso dividiendo tablas correspondientes a las plantas de consumo humano directo e indirecto que se detallan a continuación:

Tabla $\mathbf{N}^{\circ} \mathbf{8}$ Volumen en toneladas de aceites y grasas acorde al LMP vs lo estimado en plantas de consumo humano directo

\begin{tabular}{|c|c|c|c|c|c|c|}
\hline \multirow[b]{2}{*}{ EIP } & Actividades & \multirow{2}{*}{$\begin{array}{c}\text { Zona de } \\
\text { evacuación }\end{array}$} & \multirow{2}{*}{$\begin{array}{c}\text { CC. } \\
\text { Acorde al } \\
\text { LMP AyG } \\
\text { TM }\end{array}$} & \multirow{2}{*}{$\begin{array}{c}\text { CC } \\
\text { estimada } \\
\text { AyG } \\
\text { TM }\end{array}$} & \multirow{2}{*}{$\begin{array}{c}\text { Diferencia } \\
\text { de CC } \\
\text { TM }\end{array}$} & \multirow{2}{*}{$\begin{array}{c}\text { Decisión } \\
\text { TM }\end{array}$} \\
\hline & CHD & & & & & \\
\hline Planta A & Congelado & Submareal & 19.9 & 1.36 & 18.54 & Autorizado \\
\hline Planta B & Congelado-enlatado & Submareal & 32.4 & 0.92 & 31.48 & Autorizado \\
\hline Planta C & Enlatado & Submareal & 1.4 & 0.12 & 1.28 & Autorizado \\
\hline Planta D & Enlatado & Submareal & 1.13 & 0.02 & 1.11 & Autorizado \\
\hline Planta E & Enlatado-harina residual & Intermareal & 1.51 & 6.49 & -4.98 & To Autorizadı \\
\hline Planta F & Enlatado-Harina residual & Intermareal & 6.06 & 4.98 & 1.08 & Autorizado \\
\hline Planta G & Enlatado-Harina residual & Intermareal & 3.46 & 98.78 & -95.32 & Jo Autorizac \\
\hline
\end{tabular}

CC: Carga contaminante

Fuente: Elaboración propia 
Tabla $\mathbf{N}^{\circ} \mathbf{9}$ Volumen en toneladas de sólidos suspendidos acorde al LMP vs lo estimado en plantas de consumo humano directo

\begin{tabular}{|c|c|c|c|c|c|c|}
\hline \multirow[b]{2}{*}{ EIP } & Actividades & \multirow{2}{*}{$\begin{array}{c}\text { Zona de } \\
\text { evacuación }\end{array}$} & \multirow{2}{*}{$\begin{array}{c}\text { CC. Acorde } \\
\text { al LMP } \\
\text { SST } \\
\text { TM }\end{array}$} & \multirow{2}{*}{$\begin{array}{c}\text { CC } \\
\text { estimada } \\
\text { SST } \\
\text { TM }\end{array}$} & \multirow{2}{*}{$\begin{array}{c}\text { Diferencia } \\
\text { de CC } \\
\text { TM }\end{array}$} & \multirow{2}{*}{$\begin{array}{c}\text { Decisión } \\
\text { TM }\end{array}$} \\
\hline & CHD & & & & & \\
\hline Planta A & Congelado & Submareal & 39.79 & 6.78 & 33.01 & Autorizado \\
\hline Planta B & Congelado-enlatado & Submareal & 64.81 & 11.08 & 53.73 & Autorizado \\
\hline Planta C & Enlatado & Submareal & 2.80 & 0.89 & 1.91 & Autorizado \\
\hline Planta D & Enlatado & Submareal & 2.25 & 0.08 & 2.17 & Autorizado \\
\hline Planta E & Enlatado-harina residual & Intermareal & 3.02 & 27.8 & -24.78 & No Autorizad \\
\hline Planta F & Enlatado-Harina residual & Intermareal & 12.12 & 10.84 & 1.28 & Autorizado \\
\hline Planta $\mathrm{G}$ & Enlatado-Harina residual & Intermareal & 6.92 & 85.32 & -78.4 & No Autorizas \\
\hline
\end{tabular}

CC: Carga contaminante

Fuente: Elaboración propia

Las concentraciones expresadas en carga contaminante en función a la recepción de materia prima en el periodo establecido, para las actividades de consumo humano directo, según la tabla 8 para los aceites y grasas las plantas E y G estarían evacuando un excedente de $4.98 \mathrm{tm}$ y $95.32 \mathrm{tm}$, respecto a los sólidos suspendidos totales de igual manera las citadas plantas evacuan un excedente de $24.78 \mathrm{tm}$ y 78.4 tm lo que es indicador de falta de tratamiento.

\subsection{Resultados de acuerdo al objetivo 3: Identificar los parámetros físicos, químicos} y microbiológicos en la zona intermareal y submareal del agua de mar superficial que excedan los estándares de calidad ambiental donde influye la presencia de los efluentes industriales pesqueros en la Bahía de Coishco-Perú, durante el periodo de abril, junio y noviembre del año 2018.

a) Resultados de los valores promedio de acuerdo a su categoría establecida en el ECA-agua 2017

En las siguientes tablas se muestran los resultados promedios correspondientes a las categorías Categoría 1 - subcategoría B: aguas superficiales destinadas a la recreación, $\mathrm{B} 1$ : contacto primario $(\mathrm{C} 1: \mathrm{B} 1)$ y Categoría 2 subcategoría $\mathrm{C} 3$ : actividades marino portuarias, industriales, o de saneamiento en aguas marino costeras (C2:C3). Se debe indicar que para aquellos resultados donde se cuenta en los resultados del informe de ensayo con el símbolo “< al límite de cuantificación de laboratorio” se redondeó al inmediato inferior para poder realizar el promedio. 
Tabla $\mathbf{N}^{\circ} 9$ Resultados promedio de valores comparados con la categoría C1:B1 del ECAAGUA

\begin{tabular}{lcccccc}
\hline \multicolumn{1}{c}{ Parámetro } & $\begin{array}{c}\text { ECA-Agua } \\
\text { C1:B1 }\end{array}$ & AM-CO1 & AM-CO2 & AM-CO3 & AM-CO4 & AM-COS \\
\hline DBO $(\mathrm{mg} / \mathrm{L})$ & $\mathbf{5}$ & 1.99 & 3.33 & 250 & 1.99 & 1.99 \\
\hline DQO $(\mathrm{mg} / \mathrm{L})$ & $\mathbf{3 0}$ & $\mathbf{9 3 . 8 0}$ & $\mathbf{3 9 . 1 0}$ & $\mathbf{5 5 . 3 5}$ & $\mathbf{1 1 0 . 0 5}$ & $\mathbf{7 9 . 5 5}$ \\
\hline O.D $(\mathrm{NMP} / 100 \mathrm{~mL})$ & $\mathbf{5 5}$ & 7.96 & 8.13 & $\mathbf{8 . 6 9}$ & 9.13 & 5.15 \\
\hline C.T. $(\mathrm{mg} / \mathrm{L})$ & $\mathbf{2 0 0}$ & $\mathbf{4 3 0 . 3 3}$ & $\mathbf{4 8 0 3 3 3 . 3 3}$ & $\mathbf{1 9 5 0 0 . 0 0}$ & $\mathbf{5 8 4 5 . 6 7}$ & 65.60 \\
\hline AyG & $\begin{array}{c}\text { Ausencia de } \\
\text { película } \\
\text { visible }\end{array}$ & 0.78 & 0.22 & 29.54 & 0.09 & 0.09 \\
\hline SST & & 29.00 & 546.00 & 187.50 & 41.00 & 7.00 \\
\hline
\end{tabular}

Fuente: Elaboración propia

Tabla $\mathbf{N}^{\circ} \mathbf{1 0}$ Resultados promedio de valores comparados con la categoría C2:C3 del ECA-AGUA

\begin{tabular}{lccccccc} 
Parámetro & $\begin{array}{c}\text { ECA- } \\
\text { Agua } \\
\text { C2:C3 }\end{array}$ & $\begin{array}{c}\text { AM- } \\
\text { CO6 }\end{array}$ & $\begin{array}{c}\text { AM- } \\
\text { CO7 }\end{array}$ & $\begin{array}{c}\text { AM- } \\
\text { CO8 }\end{array}$ & $\begin{array}{c}\text { AM- } \\
\text { CO9 }\end{array}$ & $\begin{array}{c}\text { AM- } \\
\text { CO10 }\end{array}$ & $\begin{array}{c}\text { AM- } \\
\text { CO11 }\end{array}$ \\
\hline DBO $(\mathrm{mg} / \mathrm{L})$ & $\mathbf{1 0}$ & $\mathbf{1 0 . 3 3}$ & 1.99 & 1.99 & 1.99 & 1.99 & 1.99 \\
\hline DQO $(\mathrm{mg} / \mathrm{L})$ & & 141.35 & 107.75 & 96.95 & 85.30 & 114.95 & 137.80 \\
\hline O.D $(\mathrm{NMP} / 100 \mathrm{~mL})$ & $\mathbf{2 . 5}$ & 4.14 & 7.92 & 5.96 & 7.02 & 5.94 & $\mathbf{1 . 5 3}$ \\
\hline C.T. $(\mathrm{mg} / \mathrm{L})$ & $\mathbf{2 0 0}$ & $\mathbf{4 6 0 0 3 . 4 0}$ & 104.50 & 113.05 & 69.60 & 5.80 & $\mathbf{1 7 5 2 . 0 0}$ \\
\hline AyG & $\mathbf{2}$ & $\mathbf{2 . 8 6}$ & 0.09 & 0.75 & 0.09 & 0.09 & 0.15 \\
\hline SST & $\mathbf{7 0}$ & 28.00 & 15.00 & 12.33 & 7.67 & 6.33 & 9.00 \\
\hline
\end{tabular}

Fuente: Elaboración propia

Respecto a la tabla 9, correspondiente a la categoría C1:B1; las tomas de los puntos AM$\mathrm{CO} 1$ al AM-CO4 se realizaron en la orilla de playa (zona intermareal) y el punto AMCO5 fue realizado en la zona submareal, lo más resaltante de estos promedios que en la gran mayoría de resultados la DQO y CT superan el ECA acorde a la categoría comparada. Asimismo, teniendo como comparativo la categoría C2:C3, se puede observar que en algunos puntos exceden los parámetros DBO, AyG y CT tal como se observa en la tabla 10 . 
Tabla $\mathbf{N}^{\circ} 11$ Resultados de monitoreo de agua para la C4:C3 (noviembre/2018)

\begin{tabular}{|c|c|c|c|}
\hline Parámetro & Unidad & $\begin{array}{c}\text { Punto de } \\
\text { monitoreo } \\
\text { AM-CO12 }\end{array}$ & $\begin{array}{c}\text { ECA para agua D.S N } \mathrm{N}^{\circ} 004-2017- \\
\text { MINAM } \\
\text { Categoría } 4 \text { Conservación del medio } \\
\text { Acuático } \\
\text { Sub categoría C3 ecosistemas costeros } \\
\text { y marinos }\end{array}$ \\
\hline $\mathrm{pH}$ & (Und pH) & 7,69 & $6,8-8,5$ \\
\hline Oxígeno disuelto (OD) & $(\mathrm{mg} / \mathrm{L})$ & 7,36 & $\geq 4$ \\
\hline Aceites y Grasas (AyG) & $(\mathrm{mg} / \mathrm{L})$ & $<0,100$ & 5 \\
\hline Demanda Bioquímica de Oxigeno (DBO5) & $(\mathrm{mg} / \mathrm{L})$ & $<2$ & 10 \\
\hline Sólidos Suspendidos Totales (SST) & $(\mathrm{mg} / \mathrm{L})$ & 10 & $\leq 30$ \\
\hline Coliformes termotolerantes (CT) & $\mathrm{NMP} / 100 \mathrm{~mL}$ & $<1,8$ & 2000 \\
\hline
\end{tabular}

Fuente: Elaboración propia

En el punto AM-CO12 solo se ejecutó en noviembre del 2018 el monitoreo de agua de mar, en dicho punto es la zona en blanco donde no hay influencia de actividades antrópicas.

De los siete (7) parámetros realizados ninguno ha transgredió el valor del ECA C4:C3, tal como se detalle en la tabla 11.

\subsection{Prueba Hipótesis}

3.4.1. Nivel de concentración de los efluentes industriales pesqueros que se vierten en la zona intermareal y submareal que sobrepasan los Límites Máximos Permisibles del sector industrial pesquero o Ptar doméstica que superen el 50\%, son indicadores de gran impacto en la Bahía de Coishco-Perú, durante el periodo de febrero a noviembre del año 2018.

- La prueba de hipótesis se realiza para cada uno de los parámetros: DB05, DQO, AyG, $\mathrm{SST}, \mathrm{pH}$ y CT, tanto en aquellas empresas que vierten en la zona intermareal como en la zona submareal.

- Se usa la prueba binomial, en virtud a que las variables (indicadores se ha dicotomizado según el LMP (un grupo indica los valores no sobrepasan los LMP y el otro indica los valores sobrepasan los LMP)

- La hipótesis nula del estadístico de contraste:

Ho: $\mathrm{P}($ Si sobrepasan LMP $)=\mathrm{P}($ No sobrepasan LMP $)$

Nivel de decisión: si p-valor $<0.05$ se rechaza Ho, caso contrario no se puede rechazar Ho. 
Tabla $\mathbf{N}^{\circ}$ 11. Prueba binomial para las variables: DBO5, DQO, A y G, SST, pH y CT en la zona intermareal.

\begin{tabular}{|c|c|c|c|c|c|c|}
\hline & & Categoría & $\mathbf{N}$ & $\begin{array}{c}\text { Prop. } \\
\text { observada }\end{array}$ & $\begin{array}{l}\text { Prop. } \\
\text { De } \\
\text { prueba }\end{array}$ & $\begin{array}{c}\text { Significación } \\
\text { exacta } \\
\text { (bilateral) }\end{array}$ \\
\hline \multirow{2}{*}{$\begin{array}{l}\text { DBO5: Sobrepasan LMP } \\
\text { (PTAR doméstica) }\end{array}$} & Grupo 1 & $\mathrm{Si}$ & 48 & 1.00 & .50 & .000 \\
\hline & Total & & 48 & 1.00 & & \\
\hline \multirow{2}{*}{$\begin{array}{l}\text { DQO: Sobrepasan LMP } \\
\text { (PTAR doméstica) }\end{array}$} & Grupo 1 & $\mathrm{Si}$ & 30 & 1.00 & .50 & .000 \\
\hline & Total & & 30 & 1.00 & & \\
\hline \multirow{3}{*}{$\begin{array}{l}\text { A y G: Sobrepasan LMP } \\
\text { (PTAR doméstica) }\end{array}$} & Grupo 1 & $\mathrm{Si}$ & 21 & .44 & .50 & .471 \\
\hline & Grupo 2 & No & 27 & .56 & & \\
\hline & Total & & 48 & 1.00 & & \\
\hline \multirow{3}{*}{$\begin{array}{lc}\text { SST: } & \text { Sobrepasan } \\
\text { LMP(Sector } & \text { pesquero) }\end{array}$} & Grupo 1 & $\mathrm{Si}$ & 25 & .52 & .50 & .885 \\
\hline & Grupo 2 & No & 23 & .48 & & \\
\hline & Total & & 48 & 1.00 & & \\
\hline \multirow{3}{*}{$\begin{array}{l}\text { pH: Sobrepasan (Sector } \\
\text { pesquero) }\end{array}$} & Grupo 1 & No & 45 & .94 & .50 & .000 \\
\hline & Grupo 2 & $\mathrm{Si}$ & 3 & .06 & & \\
\hline & Total & & 48 & 1.00 & & \\
\hline \multirow{2}{*}{$\begin{array}{l}\text { CT: Sobrepasan LMP } \\
\text { (PTAR doméstica) }\end{array}$} & Grupo 1 & $\mathrm{Si}$ & 18 & 1.00 & .50 & .000 \\
\hline & Total & & 18 & 1.00 & & \\
\hline
\end{tabular}

Fuente: Elaboración propia con SPSS

Tabla $\mathbf{N}^{\circ} 12$ Prueba binomial para las variables: DBO5, DQO, A y G, SST, pH y CT en la zona submareal

\begin{tabular}{|c|c|c|c|c|c|c|}
\hline & & Categoría & $\mathbf{N}$ & $\begin{array}{c}\text { Prop. } \\
\text { observada }\end{array}$ & $\begin{array}{l}\text { Prop. } \\
\text { De } \\
\text { prueba }\end{array}$ & $\begin{array}{c}\text { Significación } \\
\text { exacta } \\
\text { (bilateral) }\end{array}$ \\
\hline \multirow{3}{*}{$\begin{array}{l}\text { DBO5: Sobrepasan LMP } \\
\text { (PTAR doméstica) }\end{array}$} & Grupo 1 & $\mathrm{Si}$ & 67 & .96 & .50 & .000 \\
\hline & Grupo 2 & No & 3 & .04 & & \\
\hline & Total & & 70 & 1.00 & & \\
\hline \multirow{3}{*}{$\begin{array}{l}\text { DQO: Sobrepasan LMP } \\
\text { (PTAR doméstica) }\end{array}$} & Grupo 1 & No & 7 & .15 & .50 & .000 \\
\hline & Grupo 2 & $\mathrm{Si}$ & 39 & .85 & & \\
\hline & Total & & 46 & 1.00 & & \\
\hline \multirow{3}{*}{$\begin{array}{l}\text { AyG: Sobrepasan LMP } \\
\text { (PTAR doméstica) }\end{array}$} & Grupo 1 & No & 69 & .99 & .50 & .000 \\
\hline & Grupo 2 & $\mathrm{Si}$ & 1 & .01 & & \\
\hline & Total & & 70 & 1.00 & & \\
\hline \multirow{3}{*}{$\begin{array}{l}\text { SST: Sobrepasan LMP } \\
\text { (Sector pesquero) }\end{array}$} & Grupo 1 & No & 60 & .86 & .50 & .000 \\
\hline & Grupo 2 & $\mathrm{Si}$ & 10 & .14 & & \\
\hline & Total & & 70 & 1.00 & & \\
\hline \multirow{3}{*}{$\begin{array}{l}\text { pH: Sobrepasan (Sector } \\
\text { pesquero) }\end{array}$} & Grupo 1 & No & 66 & .94 & .50 & .000 \\
\hline & Grupo 2 & $\mathrm{Si}$ & 4 & .06 & & \\
\hline & Total & & 70 & 1.00 & & \\
\hline \multirow{3}{*}{$\begin{array}{l}\text { CT: Sobrepasan LMP } \\
\text { (PTAR doméstica) }\end{array}$} & Grupo 1 & No & 10 & .33 & .50 & .099 \\
\hline & Grupo 2 & $\mathrm{Si}$ & 20 & .67 & & \\
\hline & Total & & 30 & 1.00 & & \\
\hline
\end{tabular}

Fuente: Elaboración propia con SPSS 


\subsection{Presentación de resultados}

\section{a) Interpretación de prueba de Prueba de hipótesis 1}

Siendo el p-valor para las variables DBO5, DQO, pH y CT menor que 0,05, se rechaza la hipótesis nula de igualdad entre las proporciones de ambos grupos, es decir, la diferencia que existe entre la proporción del grupo que si sobrepasan los LMP (indicadores de impacto) y la proporción del grupo que no sobrepasan los LMP es estadísticamente significativa.

Con respecto a la A y G y SST, se observa que el p-valor para las dos variables es mayor que 0.05 , por lo cual no se puede rechazar la hipótesis nula, es decir, la diferencia entre las proporciones de ambos grupos no es estadísticamente significativa, por ende, estos indicadores de impacto, no son considerados como indicadores de gran impacto.

En consecuencia, con un nivel de confianza del $95 \%$ se puede afirmar que en la zona intermareal el DBO5, DQO y CT, son indicadores de gran impacto en la Bahía de Coishco-Perú.

En la tabla $\mathrm{N}^{\circ} 12$, se observa que el p-valor para las variables DBO5, DQO, A y G, SST y $\mathrm{pH}$ es menor que 0.05 , por lo tanto, se rechaza la hipótesis nula, es decir que la diferencia entre la proporción del grupo que, si sobrepasan los LMP y el grupo que no sobrepasan los LMP, es estadísticamente significativa. En la misma tabla se observa que el porcentaje del grupo si sobrepasan los LMP para las variables DBO5 y DQO superior al 50\%.

El p-valor (0.099) para la variable CT es mayor que 0.05, por lo cual no se puede rechazar la hipótesis nula, es decir, que la diferencia entre ambos grupos (los que superan y los que no superan) no es estadísticamente significativa, por ende, no se puede considerar a esta variable como indicador de gran impacto.

Resumiendo, se puede indicar que en las empresas que vierten en la zona intermareal se consideran como indicadores de gran impacto en la Bahía de Coishco-Perú a los parámetros del DBO5, DQO y CT, mientras que en las empresas que vierten en la zona submareal a los parámetros DBO5 y DQO.

\section{CONCLUSIÓN O CONSIDERACIONES FINALES}

- Al comparar los resultados promedio de las concentraciones de los parámetros Aceites y Grasas (A y G) y Sólidos Suspendidos Totales (SST), presentes en los efluentes con los Límites Máximos Permisibles (LMP) del sector pesca correspondiente al año 2018. Podemos señalar que las plantas que vierten en la Zona 
Submareal la gran mayoría cumplen con los LMP, muy distinta es la situación de las plantas que vierten en la Zona intermareal donde se observa excesos, siendo un indicador de falta de tratamiento de las aguas residuales.

- Al confrontar de manera referencial los resultados de las concentraciones promedios de los parámetros Demanda Bioquímica de Oxígeno (DBO), Demanda Química de Oxígeno (DQO) y Coliformes Termotolerantes (C.T) presentes efluentes con los LMP de PTAR doméstica, podemos afirmar que las plantas que vierten sus efluentes en la zona submareal e intermareal no alcanzaría a cumplir con la norma de referencia, esto debido a que los sistemas de tratamiento implementado no tendrían la finalidad de reducir los citados parámetros.

- Mediante el programa SPSS, se pudo determinar que las concentraciones de parámetros contaminantes cuyo número de resultados mayor al 50\% superan los LMP, catalogados como indicadores de gran impacto. Se concluye que para aquellas plantas que evacuan en la zona submareal los parámetros son la DBO y DQO y para las plantas que evacuan en la zona intermareal los indicadores de gran impacto son la DBO, DQO y C.T.

- Para la determinación del nivel de carga contaminante en función a los parámetros DBO, DQO, SST y A y G presentes en los efluentes industriales pesqueros de las actividades de consumo humano directo e indirecto, dio como resultado que la carga contaminante expresado en toneladas durante el año 2018, fue mayor para los parámetros DBO, DQO y SST para aquellas plantas que vierten en la zona submareal y para las plantas que vierten en la zona intermareal la mayor carga fue del parámetro A y G.

- Al contrastar los resultados promedio de las concentraciones de los parámetros DBO, DQO, OD, C.T, A y G y SST en la Bahía de Coishco para el año 2018, se obtuvo para la categoría C1: B1 acorde a la categoría establecida en el ECA-Agua de los cinco puntos evaluados en todos exceden el parámetro DQO y en cuatro puntos exceden el parámetro C.T, con relación a la categoría C2: C3 de los seis puntos evaluados se refiere en uno se excedió la DBO, CT y A y G y dentro de esta misma categoría un punto reportó una baja de OD y un exceso en los CT, esto posiblemente sea indicadores de actividades antrópicas y domésticas cercanas a la Bahía. 
- Al finalizar el presente trabajo de investigación, se proponen alternativas tecnológicas para el tratamiento de aguas con el uso de tratamientos físicos, químicos y la aplicación de ozono, esto en función de la realidad de cada planta, con la finalidad de reducir sus aportes contaminantes en la Bahía de Coishco.

\section{RECOMENDACIONES}

- Para aquellas plantas que no cumplen con los LMP del sector pesca, las autoridades competentes deben promover mediante acuerdos el cumplimiento que mediante plazos se generen mecanismos de acción e implementación de sistemas de mitigación que beneficien al ambiente.

- Respecto a los parámetros DBO, DQO y CT, si bien no existe un LMP se tomó referente el LMP de PTAR doméstico, donde se apreció que la gran mayoría de plantas no lo cumplen, por lo que es necesario que las autoridades competentes establezcan un límite para los citados parámetros tomando en cuenta la legislación nacional existente plantea su realización desde el año 2016.

- Las autoridades responsables de la fiscalización en materia ambiental en los cuerpos de agua, deben tener mayor presencia de acuerdo a sus competencias, asimismo identificar la ubicación correcta de las fuentes de vertimiento autorizadas y no autorizadas, con la finalidad que los resultados de monitoreo sean lo más fiables al momento de identificar la correcta zona de mezcla.

- Proponer a los administradores como conductores de sus plantas pesqueras y gestores del tratamiento de sus efluentes industriales pesqueros, invertir, en tecnologías amigables con el ambiente, asimismo debe romperse el paradigma empresarial que la implementación de sistemas de tratamiento para reducir los aportes contaminantes como un gasto, si no debe entenderse como una inversión que asegure competitividad en el mercado.

\section{LISTA DE REFERENCIAS}

Ambrosio, M. J. (2004). Procesamiento pesquero, dsiposición de residuos, e impacto ambiental. In. Congreso Argentino de Saneamiento y Medio Ambiente, 14, (pp. 18).

Ataner Moraga, R. A. (2009). Descripción y análisis comparativo de los sistemas de tratamiento de RIL en la industria de Alimentos en Valdivia. (Tesis de Pregrado, Universidad Austral de Chila, Valdivia, Chile. 
Aquino, M., \& Maguiña, S. (2018). Evaluación de la Contaminación Marina de la Bahía de Coishco 2015 - 2016, Ancash, Perú., (tesis de posgrado) Universidad Nacional del Santa, Ancash, Perú.

Austin Enginnering Perú S.A.C. (2013). Proceso y harina de pescado. Recuperado el 2021 de abril de 2, de https://www.slideshare.net/victoralayo/proceso-de-la-harina-depescado.

Bereche Crisanto, J.S. (2013). Evaluación de la propuesta de mejoramiento en la producción de harina residual de pota (Dosidicus giga) en la empresa Seafrost SAC - Paita 2018. (Tesis de pregrado), Universidad Nacional de Piura, Piura, Perú.

Cabrera Carranza, C. F. (2002). Estudio de la contaminación de las aguas costeras en la bahía de Chancay: propuesta de recuperación., (tesis de posgrado) Universidad Nacional Mayor de San Marcos, Lima, Perú.

CONAM-Consejo Nacional del Ambiente. (1999). Diagnóstico Ambiental de la Bahía El Ferrol (1 ed.). Lima, Perú.

Esquivel, F., Adelaida, P., \& Yalico Cavero, C. (2015). Impacto ambiental de los efluentes de la industria pesquera en las aguas de mar de la bahía de chancay., (tesis de pregrado) Universidad Nacional José Faustino Sánchez Carrión, Lima, Perú.

Grupo Banco Mundial. (2007). Corporación Financiera Internacional. Environmental, Health and Safety Guidelines for Fish Processing [Guías sobre Medio Ambiente, Salud y Seguridad para el Procesamiento de Pescado]. Obtenido de http://www.ifc.org/ifcext/sustainability.nsf/Content/EnvironmentalGuidelines

Grupo Banco Mundial. Corporación Financiera Internacional, 2007. Environmental, Health and Safety Guidelines for Fish Processing [Guías generals sobre Medio Ambiente, Salud y Seguridad]. Washington, DC: Grupo Banco Mundial. Obtenido de http://www.ifc.org/general/ehs/spanish/final/rev/cc.pdf.

INDECOPI (Instituto Nacional de Defensa de la Competencia y de la Protección de la Propiedad Intelectual) 1986. NTP.204.039:1986. Almacenamiento de Harina y Aceite de Pescado. Lima. 4p.

Instituto de Hidrografía, Meteorología y Estudios Ambientales-IDEAM, 2017. (2017). [Metodología para la Evaluación aproximada de la Carga Contaminante]. 
Guevara del Aguila...

Obtenido

de

http://documentación.ideam.gov.co/openbiblio/bvirtual/011117/Carga_Organic a.pdf.

Pesquera Miguel Angel S.A.C. (2018). Informe técnico sustentatorio para modificar la disposición final de efluentes industriales y domésticos en el establecimiento de enlatado y harina residual. Santa, Perú. $15-18 p$.

Rodríguez Guerrero, M.A (2007). Conservas de pescado y sus derivados. Edit. Ediciones Universitarias. Universidad del Valle, Cali, Colombia. 7p,

Romero López, T. D. J. (2013). Aporte contaminante del procesamiento de recursos pesqueros en Cuba y su impacto al medio. Ingeniería Hidráulica y Ambiental, 34(2), 17-26.

Rosales, H. (2012). Conservación de alimentos por calor. Huancayo, Perú.

Sánchez, G., Tam, J., \& Vera, G. (2000). Pruebas ecotoxicologicas de efluentes pesqueros para determinar la calid de agua de mar en la bahia de Paracas (Pisco-Perú). En Informe N³01, Callao.Perú,14.

Tresierra, A., García, M., Huerto, M., Berru, P., Reyes, D., \& Cervantes, C. (2007). Recursos vivos y ambiente de las bahias Coishco, El Ferrol y Samanco de la Region de Ancash, Perú 2001-20005. Imparpe, Volumen34( $\left.{ }^{\circ} 1\right)$.

Universidad Nacional del Sur-UNS. (2009). [Aspectos generales sobre la congelación del pescado]. Argentina: UNS. Recuperado el 2020 de diciembre de 20, de https://repositoriodigital.uns.edu.ar/bitstream/handle/123456789/1945/CA

PITULO\%203.pdf?sequence=7\&isAllowed $=y$

Valverde Bustamante, J. H. (2014). Análisis de Impacto Ambiental en las Plantas Procesadoras de Productos del Mar de la Empresa Sálica del Ecuador SA., (tesis de pregrado) Universidad Guayaquil, Ecuador. 